\title{
LAND COVER EXTRACTION OF COASTAL AREA FROM GF-1 WFV IMAGERY USING ONTOLOGICAL METHOD
}

\author{
H. Luo ${ }^{1,2}$, B. He ${ }^{1,2,} *$ X. Kuai ${ }^{1,2}, \mathrm{Y} \mathrm{Li}^{1,2}$, R.Z. Guo ${ }^{1,2}$ \\ ${ }^{1}$ Institution of Smart Cities, Shenzhen University, 518060, Shenzhen, China \\ ${ }^{2}$ Shenzhen Key Laboratory of Spatial Information Smart Sensing and Services, School of Architecture and Urban Planning, 518060, \\ Shenzhen University, Shenzhen, China
}

Thematic Information Extraction (III/1)

KEY WORDS: Ontology, land cover, GF-1, Wide Field of View (WFV) image, information extraction

\begin{abstract}
:
As a knowledge organization and representation method, ontology that can store land cover spectral, texture, shape attributes and relationships derived from image analysis. With the knowledge organized in ontology, the efficiency of automatic or semi-automatic land cover information extraction for the large coastal area is supposed to be improved. Together with the help of GF-1 Wide Field of View (WFV) data, which covers almost $200 \mathrm{~km}$ width area, the more frequent monitoring and change detection for coastal area of Guangxi province are available. This study makes attempt to monitor the land cover of Guangxi coastal area using GF-1 WFV data with ontological method. The land cover ontology for this area is established first via image feature analysis. Using this ontology, automatic image extraction from GF-1 WFV data of subsequent monitoring time is realized. The results of this study reveal that, using ontology, land cover extraction can be completed in acceptable accuracy but with higher efficiency.
\end{abstract}

\section{INTRODUCTION}

Remote sensing data is widely used in different application fields nowadays. For China, to achieve the annual National Geo-survey and monitoring, manual delineating land cover information from the large amount of remote sensing data is needed once a year, which covers the whole China area or even just a certain area, is an exhausting and challenging work. Therefore, a fast and effective automatic or semi-automatic method of extraction of land cover is needed.

Pixel-based image extraction has been used in different applications for quite a long time, which has the disadvantage that information contained in the image is not fully utilized and is not suitable for high resolution image ( $\mathrm{Li}, 2012)$ (Thomas, 2014) (Arvor, 2013). Object-oriented feature extraction that uses segmentation, spectral, texture, and shape information of the object solves these problems (Thomas, 2014) (Olive-Santos, 2014). It is widely used in image feature extraction in a period of time (Baraldi, 2012). However, without the organization, storage and formal representation of information and knowledge for the feature, object-oriented method has shortages in its reusability and automation, which limits its usage by nonprofessional users or in large workload mission (Thomas, 2014) (Arvor, 2013).

Using ontology in remote sensing image extraction has been discussed in GIS and remote sensing communities (Agarwal, 2005) (Thomas, 2014). Ontology allows the representation of concepts, instances, relationships, and axioms and permits the inference of implicit knowledge (Fonseca, 2002). Many studies prove that ontologies are feasible in supporting the semantic representation of remote sensing images (Forestier, 2002) (Durand, 2007) (Jensen, 2009) (Hashimoto, 2011) (Samuel, 2012). However, most of the studies have some limitations, e.g., the feature value stored for different classes in the ontology are given by expert's prior knowledge and experiences about the study area. In other words, the result highly depends on the expert's knowledge and prior experiences, which may be different when organized by different people. And what is more, these studies ignore the acquisition time of the features of land cover class. Especially the spectral feature may be different when the time or season changes (Jensen, 2009).

In National Geo-survey mission, compared with manual delineation which is still used at present, using knowledge in automation or semi-automation extraction will improve the land cove information extraction work in both efficiency and effect. Meanwhile, satellite remotely sensed image with a large swath width is required, so that the monitoring could cover more area frequently.

This study introduces an ontology-based framework and method for land cover extraction in the Beibuwan Gulf in Guangxi, China. The GF-1 Wide Field of View (WFV) data is used in this study, which could provide us with almost 200 kilometers wide multispectral image and may cover the whole Beibuwan Gulf in one image. This paper is organized into 4 sections. The first section is the introduction. In the second section, the data used in our experiment is introduced and the methods are proposed. Then results and discussions are presented in the third section. Finally, conclusions are drawn in the last section.

\section{DATA AND METHODS}

\subsection{Data}

GF-1 is the first satellite in the Chinese high resolution earth observation system. It was launched on Apr. 26, 2013, carried by the LM-2D rocket. This successful launch started the new era of earth observation of china. The GF-1 is equipped with a 2-meter resolution panchromatic camera, an 8-meter resolution multispectral camera and four 16-meter multispectral large format cameras (WFV1, WFV2, WFV3, WFV4); the swath

\footnotetext{
* Corresponding author
} 
width is up to $200 \mathrm{~km}$ with one WFV camera, and $800 \mathrm{~km}$ with four cameras together. The GF-1 has made several technical breakthroughs. They are in optical remote sensing, which has combined high spatial resolution, high temporal resolution and multispectral technics; in high load images mosaic and fusion, in high accuracy and high stability satellite attitude control, in high resolution data processing and application, and in prolonging the satellite's service life to 5-8 years. Detailed information of GF-1 satellite and its WFV camera can be seen in Table 1.

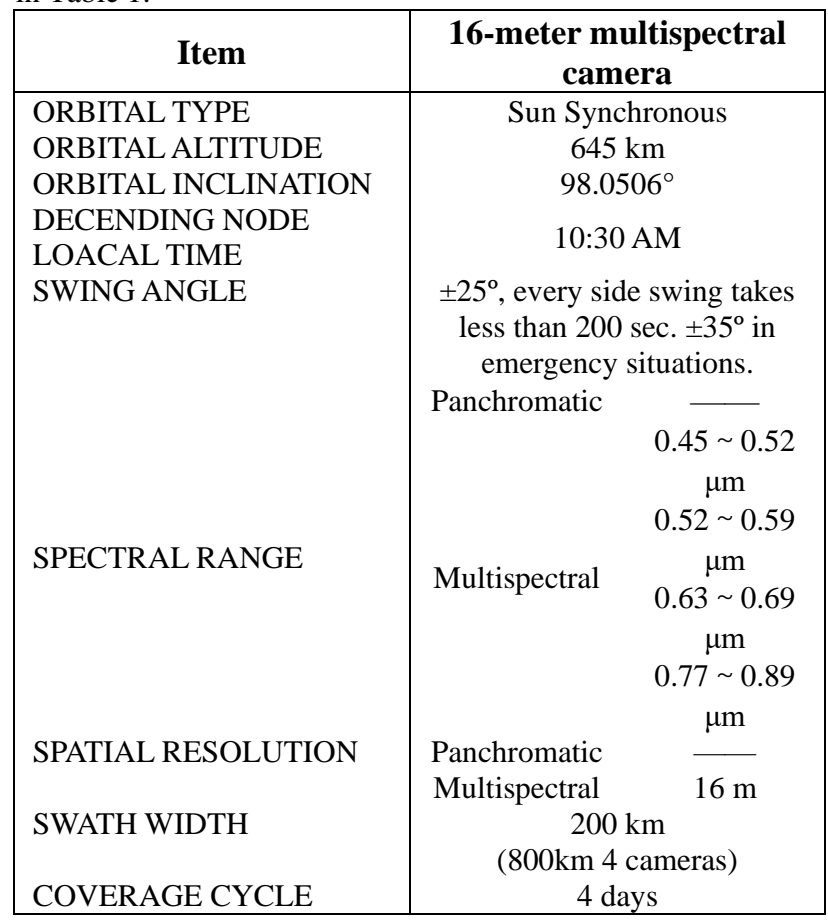

Table 1. Parameters of GF-1 satellite and its WFV camera.

The study area is part of the Beibuwan Gulf shore in Guangxi, China. This area lays in southern part of China. It is with great importance in marine economy of Guangxi, and also plays vital role in Guangxi's ecological environment and climate regulation. In this case, frequent, accurate and large scale land cover monitoring is necessary. Therefore, GF-1 WFV data is useful in our work of land cover monitoring for Beibuwan Gulf. We use the two GF-1 WFV images acquired from GF-1 WFV1 camera and WFV2 camera respectively, on April 1st, 2017.

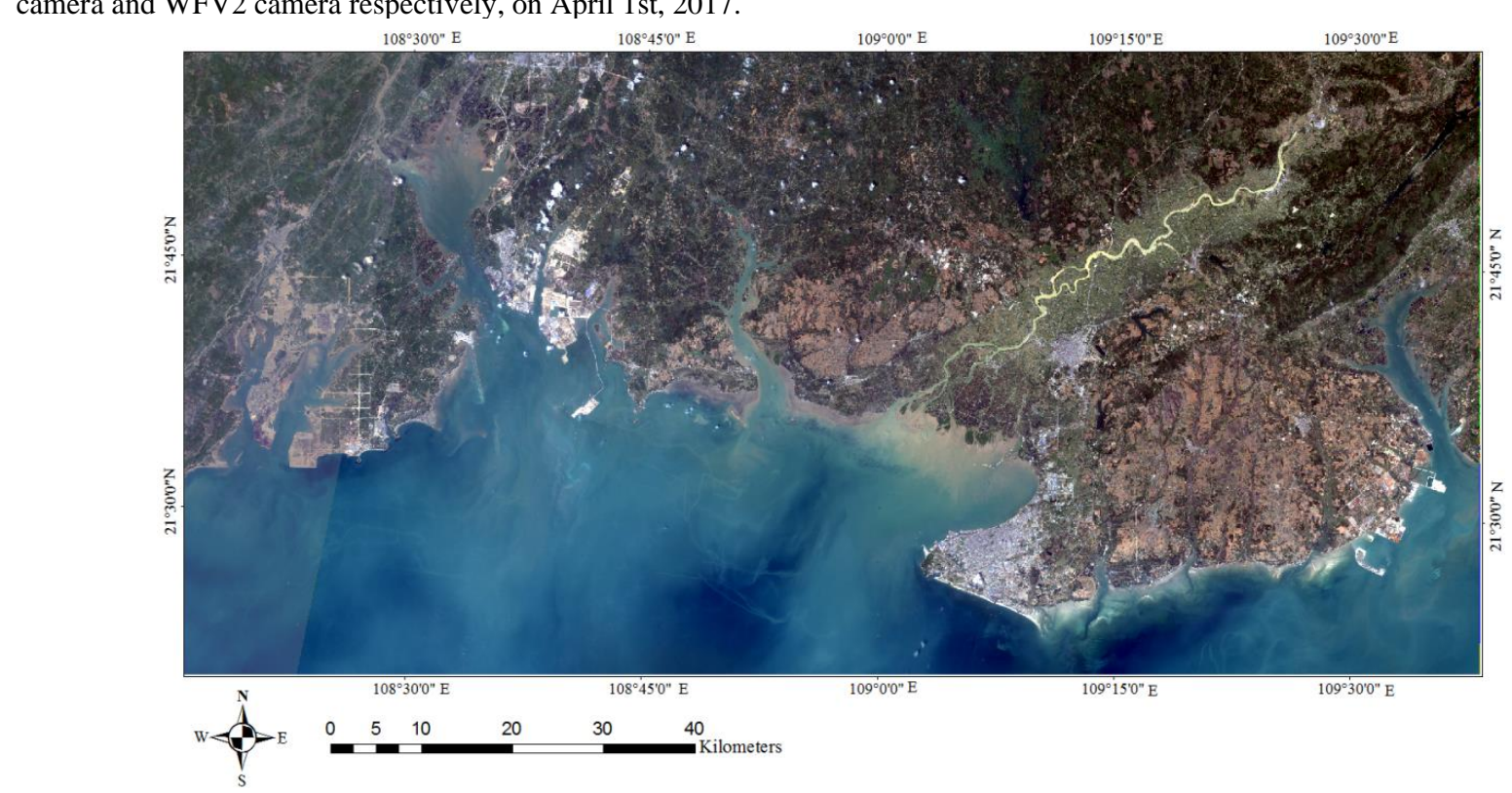

Figure 1. GF-1 WFV image of Beibuwan Gulf acquired on April 1st, 2017.
First we ortho-rectify these images using their Rational Polynomial Coefficients (RPC) models. Radiometric correction and quick atmospheric correction are done. Then these two images are mosaicked and subset of this image for our study area is clipped, which covers 140 kilometers * 60 kilometers area, with $1 \%$ cloud coverage, and good image quality. GF-1 WFV image for study area can be seen in Figure 1.

\subsection{Methods}

To achieve the ontology-based extraction of land cover in the coastal area, firstly we establish ontology with hierarchical system, classes, and properties for the land cover according to the China fundamental geographic information category used in National Geo-survey. Properties of land cover includes the spectral feature, texture feature, spatial feature, and the segmentation scale, with which the feature of land cover class on remote sensing image is formally represented.

Land cover of Beibuwan Gulf in our study are consisted of 8 types, including built-up area, cultivated land, grassland, forest, bare soil, sand, and waterbody. Framework of these land cover classes are established according to the hierarchy that the CGNCM specification provides us. Property is important for class in the ontology. Spectral, texture, spatial feature are used in ontology properties, because they are the most significant characteristics in the remote sensed image for land cover.

\subsubsection{Create land cover ontology for Beibuwan Gulf}

Hierarchy system of land cover in Beibuwan Gulf is established via Protégé. The main relationship of these classes is "is a", which builds the hierarchical structure of land cover (Fig.1). Property is important element for ontology. As mentioned above, features of land cover class in remote sensing imagery are considered as their properties, including spectral feature, texture feature, and spatial characteristic. Moreover, these properties contain more specific features, e.g., in the texture property, texture variance, entropy, mean are included, in spectral property, mean, standard deviation of different bands of the imagery are used, and area, length, elongation and so on features provide the formal presentation of spatial property. All these properties are store in Protégé as the data property.

With the help of ontology, the main framework of land cover properties and remote sensing features is formed, where prototype can be created and organized.

This contribution has been peer-reviewed. The double-blind peer-review was conducted on the basis of the full paper. https://doi.org/10.5194/isprs-annals-V-3-2020-53-2020 | @ Authors 2020. CC BY 4.0 License. 


\subsubsection{Create prototype for land cover classes}

Prototype is from the Prototype View of Concepts in Cognitive Psychology (Galotti, 2013). Study of Medin and Smith consider the concept is the abstraction of different classes, and prototype is the idealistic representation of objects or events. In other words, the prototype is the abstraction that has the centralized characteristics in all features of the objects of this class. With the help of prototype, people can classify other unknown object or event. Creating prototype of land cover class may also be of great help in image extraction. Once prototype of National Geo-survey land cover classes for one certain area in the reference year is established, it can be used in land cover extraction of the same area in subsequent year.

To create the prototype for land cover class, available ground truth and imagery of the study area in the reference year should be used. Calculate the mean value and data range of all the objects in this class, including spectral attribute, texture attribute, and spatial attribute. Storing attribute information as data properties for corresponding class in protégé, therefore, the prototype of this class is formed. Figure 2 shows the process of building land cover ontology.
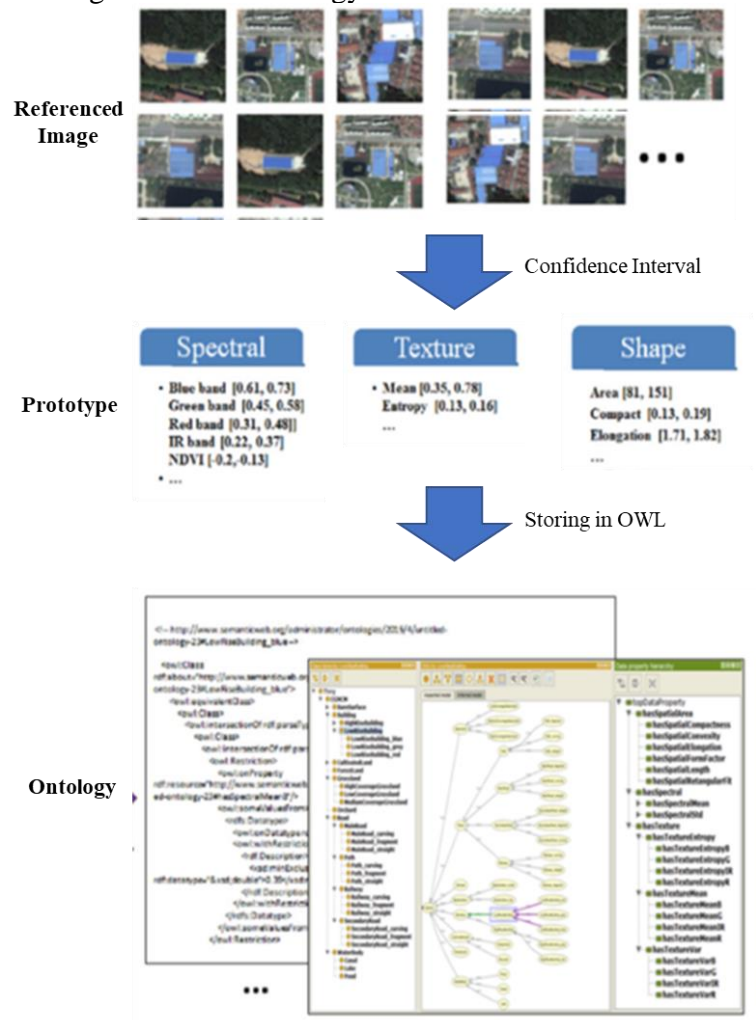

Figure 2. Create prototype of Land cover for Beibuwan Gulf. With the help of referenced image and land cover map, the confidential interval of each feature can be calculated. And then all these data are stored in Protégé 3.4.7.

\subsubsection{Ontology-based Land Cover Extraction}

Once we create the ontology and store the prototype for certain area and certain time, the land cover classes in this area can be extracted automatically via program in the same time or season of every year afterward.

Based on the ontology and prototype of land cover in Beibuwan Gulf, with the help of ENVI/IDL, the images of this area acquired in subsequent years are input, and program loads the classes of land cover from the ontology into the rule-based extraction module. Meanwhile, the data property values of land cover class prototype are used as the values of corresponding attributes of different features of classes in the program. Figure 3 shows the workflow of our study.

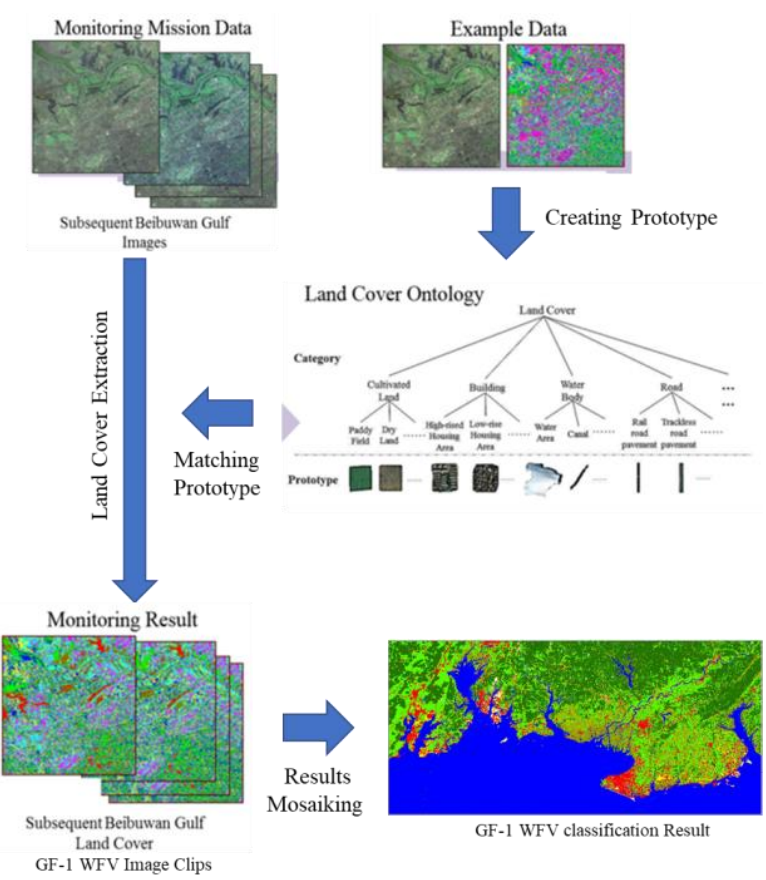

Figure 3. Procedure of using ontology and prototype in land cover extraction for Beibuwan Gulf. With the help of prototype, which is created from the example data, the data ranges of features are inputed into the extraction procedure, and then automatic land cover extraction for subsequent image can be done.

\section{RESULTS AND DISCUSSIONS}

Firstly, this study creates a hierarchical structure for land cover of Beibuwan Gulf, which is suitable for land cover extraction. In some studies, segmentation accuracy causes the limitation of object-oriented extraction for remote sensing imagery. Indeed, as the result of image quality, image preprocessing method, and the complexity of ground object, no segmentation method can be completely suitable for all kinds of land cover classes. To avoid this limitation, in this study, some of the land cover classes are divided into subclasses or components in ontology, thus these classes can be better detected in computer. And then after the extraction, subclasses or components are combined back to their parent class.

To create the land cover ontology prototype for Beibuwan Gulf area, GF-1 WFV imagery and land use and land cover map are used for present a formalization of the knowledge of the expert in land cover extraction.

From the results of reference classification map, the mean feature values of each object in different classes are calculated, and with which the prototype of each class is formed. We store the knowledge in the form of values in the data properties of land cover ontology. The so called prototype of the land cover class is then created and stored in OWL file.

We use ENVI/IDL in land cover extraction. The segmentation scale is 40 , and merge scale is 85 in this study. These two parameters will be used in the subsequent image extraction. The rule-based classifier of ENVI EX 4.8 is used in this study, and confidence threshold value is 0.40 , and default fuzzy tolerance value is set to be $5 \%$. S-Type is used as the default membership function set type. 
In order to evaluate the quality of this interpretation, we used the ground truth provided by the expert to compute confusion. The Kappa coefficient of the experiment is 0.47 , and the overall accuracy is 0.80 . The results can be seen in Table. 2 .

\begin{tabular}{|l|cc|}
\hline \multicolumn{1}{|c|}{ Class } & Prod. Acc. & User Acc. \\
\hline Sand & $77.61 \%$ & $73.45 \%$ \\
Grassland & $76.48 \%$ & $88.23 \%$ \\
Cultivated Land & $86.39 \%$ & $80.53 \%$ \\
Forest & $82.36 \%$ & $64.76 \%$ \\
Built-up Area & $85.73 \%$ & $83.88 \%$ \\
Bare Soil & $78.02 \%$ & $46.00 \%$ \\
Waterbody & $82.86 \%$ & $94.95 \%$ \\
\hline Overall Acc. & \multicolumn{2}{|c|}{$80.11 \%$} \\
\hline
\end{tabular}

\section{Table. 2 Accuracy of the results}

The results show that, proposed ontology-based image extraction method has high accuracy of extracting built-up area, cultivated land, grassland, waterbody, but low accuracy in classifying the forest, and soil. Especially, bare soil has lowest user accuracy of 0.46 , because some of the bare soil objects may be confused by built-up area or cultivated land. Figure 5, Figure 6, and Figure 7 clearly show the extraction results. As we can see, most of the land cover objects are correctly extraction.

Though the Kappa value is average, this ontology-based method is still useful in Beibuwan Gulf land cover monitoring, for it can provide the information extraction results fast and widely, and after some post classification processing, accuracy would be further improved. By this means, the manual work of land cover extraction is greatly reduced. Moreover, the method of this study provides another possibility that remote sensing information extraction knowledge can be formalized and represented, thus the knowledge would be shared and reused for other applications.
In future work, more land cover examples would be collected and used for improving the statistics for prototype features. More classifiers would be added or substituted for the classifiers that result worse accuracy, for the purpose of enhancing the extraction results. Moreover, some more attributes and complex relationships of the land cover classes would be considered. Therefore, objective features of the different applications or different area may be matching or be relating with the existing knowledge.

\section{CONCLUSIONS}

Our study proposes an ontology-based image extraction method for land cover in Beibuwan Gulf shore in Guangxi, China. The land cover ontology is first established for study area, including spectral, texture, and shape properties. Referenced land cover map and GF-1 WFV image are then used to create a land cover regional prototype for the study area, which is stored in an OWL file. Land cover extraction experiment is then conducted for the study area the year after the referenced year.

Results of this study shows that the use of ontology can help to organize land cover extraction knowledge, avoiding the variance and subjectivity caused introduced by different remote sensing experts. And automation extraction with accepted accuracy could be done according to the established ontology.

The main contribution of this study is the attempt to use ontological method and prototype for land cover extraction in coastal area like Beibuwan Gulf shore. This method has the advantage of reusability of knowledge of land cover remotely sensed characteristics, and it provides a more automatic, more efficient, and less expert knowledge-dependent way for land cover monitoring.

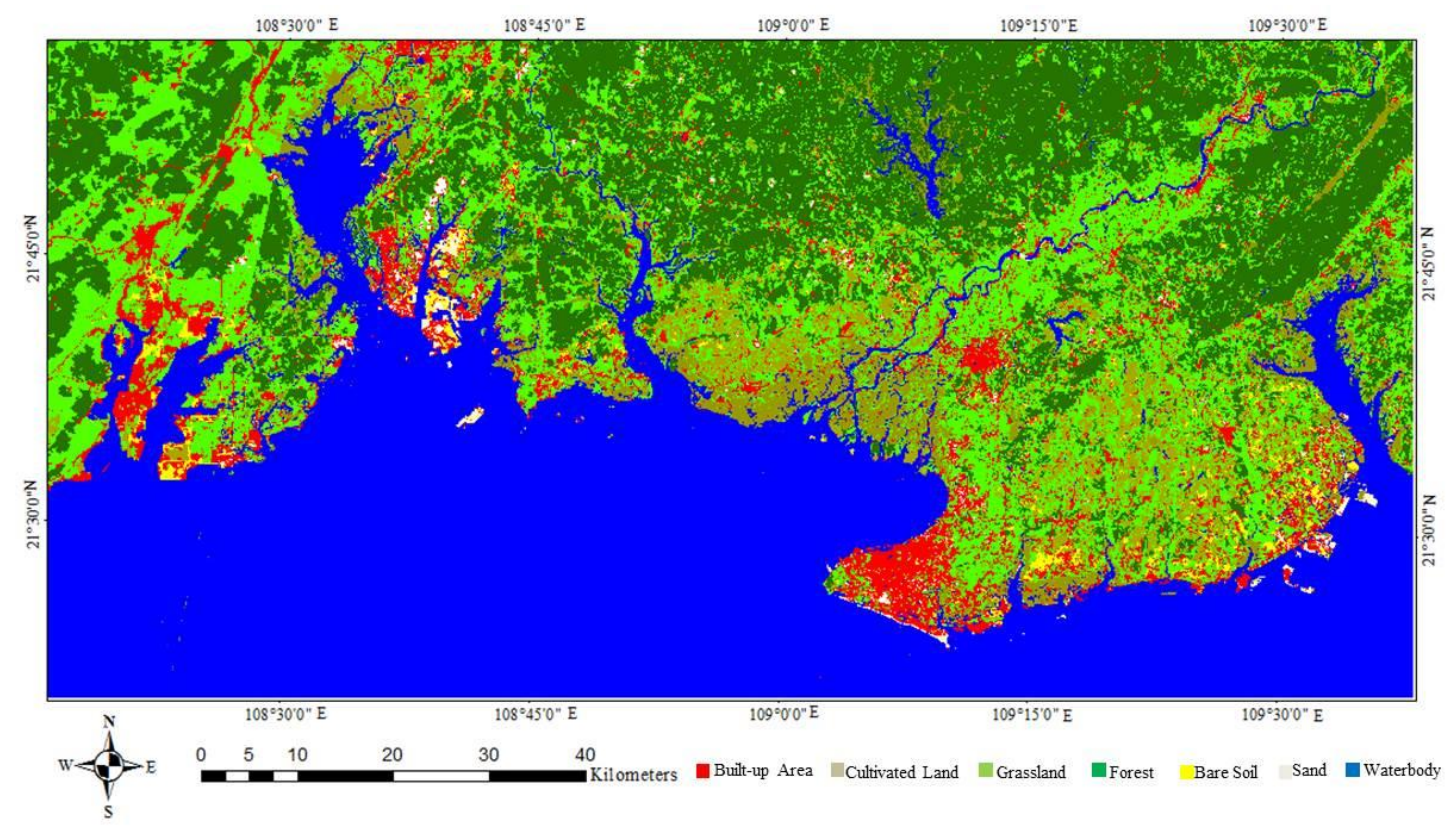

Figure 4. Extraction results of land cover for Beibuwan Gulf in 2017. 


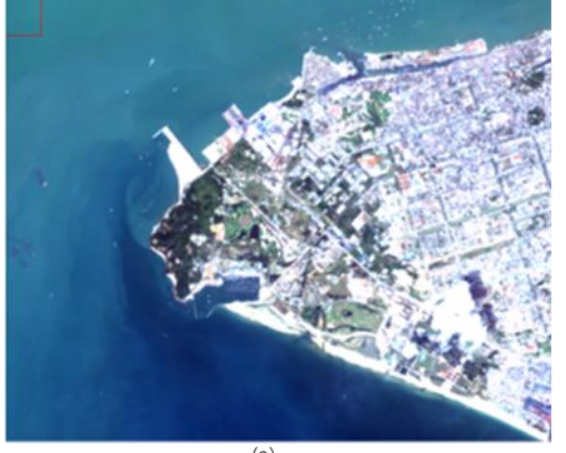

(a)

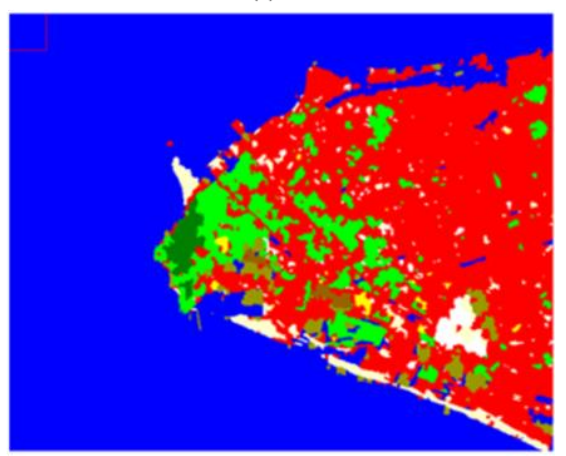

(b)

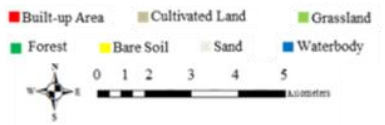

Figure 5. Clips of land cover extraction results for Beibuwan Gulf peninsula in 2017.

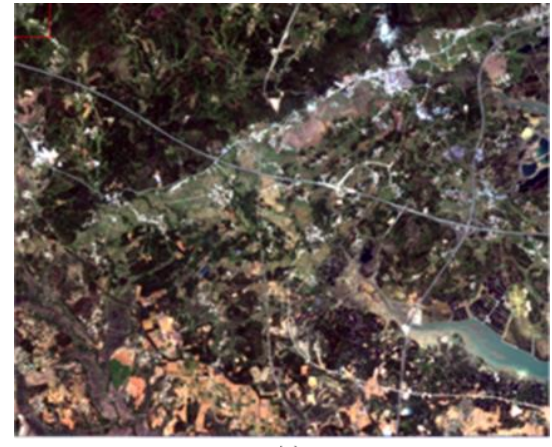

(a)

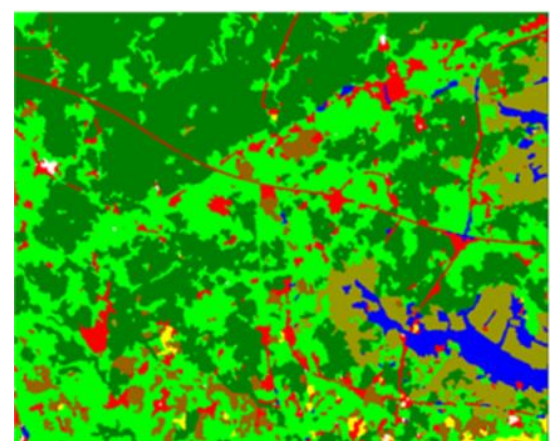

(b)

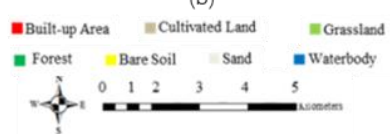

Figure 6. Clips of land cover extraction results for Beibuwan Gulf inner area in 2017.

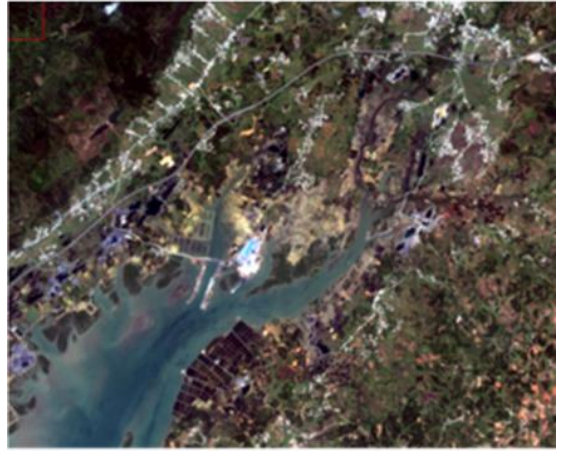

(a)
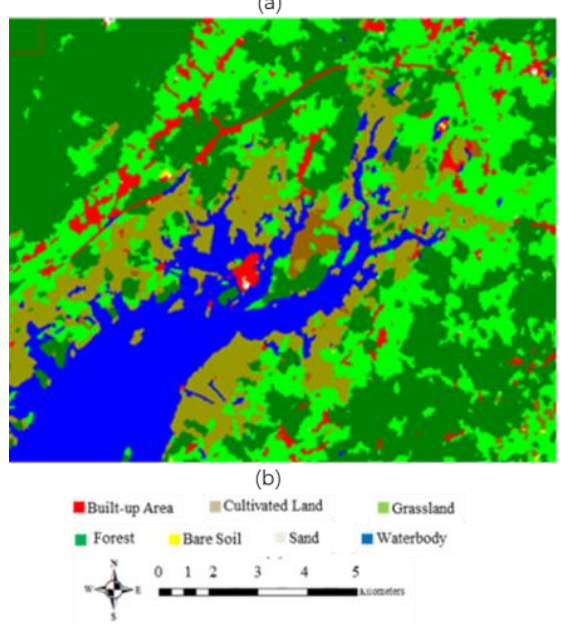

Figure 7. Clips of land cover extraction results for Beibuwan Gulf seagulf area in 2017.

\section{REFERENCES}

Agarwal, P. Ontological considerations in GIScience. International Journal of Geographical Information Science 19.5 (2005): 501-536.

Arvor, D., Durieux, L., Andres, S. et al. Advances in geographic object-based image analysis with ontologies: a review of main contributions and limitations from a remote sensing perspective. ISPRS Journal of Photogrammetry and Remote Sensing 82 (2013): 125-137.

Baraldi, A., Luigi, B. Operational automatic remote sensing image understanding systems: Beyond geographic object-based and object-oriented image analysis (GEOBIA/GEOOIA). Part 1: Introduction. Remote Sensing 4.9 (2012): 2694-2735.

Durand, N. et al. Ontology-based object recognition for remote sensing image interpretation. Tools with Artificial Intelligence, 2007. ICTAI 2007. 19th IEEE International Conference on. Vol. 1. IEEE, 2007.

Fonseca, F.T. et al. Using ontologies for integrated geographic information systems. Transactions in GIS 6.3 (2002): 231-257.

Forestier, G. et al. Knowledge-based region labeling for remote sensing image interpretation. Computers, Environment and Urban Systems 36.5 (2012): 470-480.

Hashimoto, S., Tadono,T. et al. A framework of ontology-based knowledge information processing for change detection in remote sensing data. Geoscience and Remote Sensing Symposium (IGARSS), 2011 IEEE International. IEEE, 2011.

Jensen, J.R. Remote Sensing of the Environment: An Earth Resource Perspective 2/e. Pearson Education India, 2009.

Li D.R., Sui H.G., and Jie S. Discussion on key technologies of geographic national conditions monitoring. Geomatics and Information Science of Wuhan University 37.5 (2012): 505-512. 
Li, L. Luo, H. et al. User-Oriented Image Quality Assessment of ZY-3 Satellite Imagery. IEEE Journal of Selected Topics in Applied Earth Observations and Remote Sensing 7.11(2014): 4601-4609.

Medin, D.L. Smith, E.E. Concepts and concept formation. Annual Review of Psychology, 35(1984): 113-138.

Oliva-Santos, R. et.al. Ontology-based topological representation of remote-sensing images. International Journal of Remote Sensing 35.1 (2014): 16-28.

Samuel, A., Arvor, D. and Pierkot. C. Towards an ontological approach for classifying remote sensing images. Signal Image Technology and Internet Based Systems (SITIS), 2012 Eighth International Conference on. IEEE, 2012.

The cloud service platform of ZY-3 satellite image. http://cloud.satimage.cn/EN/data_intro. (May 1st, 2017).

Galotti, K.M. Cognitive psychology in and out of the laboratory. SAGE, 2013.

Thomas B., et al. Geographic Object-Based Image AnalysisTowards a new paradigm. ISPRS Journal of Photogrammetry and Remote Sensing 87 (2014): 180-191.

Huang, H., Chen, J., Li, Z., Gong, F. and Chen, N. OntologyGuided Image Interpretation for GEOBIA of High Spatial Resolution Remote Sense Imagery: A Coastal Area Case Study. ISPRS International Journal of Geo-Information, 6.4(2017):105-122. 